
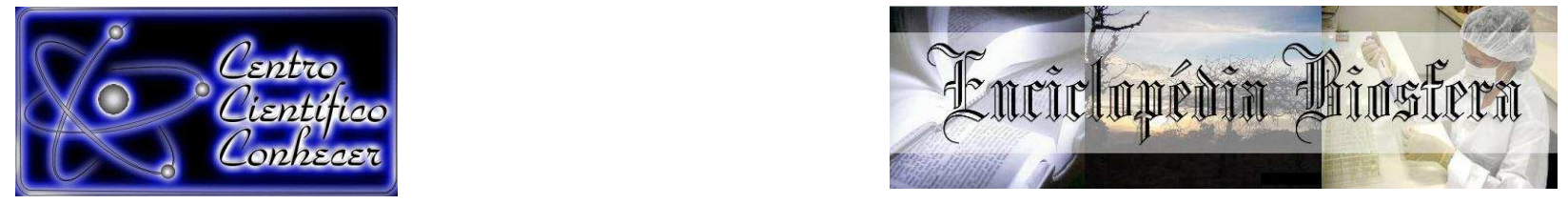

\title{
PERFURAÇÃO OCULAR EM JAGUATIRICA (Leopardus pardalis): RELATO DE CASO
}

Cleiton Azevedo $^{1}$, Deusdete Conceicão Gomes Junior ${ }^{*}$, Débora Passos Hinojosa Schaffer ${ }^{3}$, Ana Cláudia Santos Raposo ${ }^{4}$, Nayone Lima Lantyer Cordeiro de Araujo ${ }^{4}$,

1. Programa de pós graduação em Oftalmologia Veterinária, Anclivepa - SP.

2. Professor Adjunto do Campus Multidisciplinar de Barra, Universidade Federal do Oeste da Bahia (UFOB). (deusdete.gomes@ufob.edu.br); Av. 23 de agosto, Barra - Ba. CEP. 47100.000.

3. União Metropolitana para Educação e Cultura (UNIME, Lauro de Freitas)

4. Escola de Medicina Veterinária e Zootecnia. Universidade Federal da Bahia (UFBA).

Recebido em: 02/10/2017 - Aprovado em: 21/11/2017 - Publicado em: 05/12/2017 DOI: 10.18677/EnciBio_2017B47

\begin{abstract}
Foi resgatada na região de Feira de Santana, Bahia, uma jaguatirica com laceração cutânea e perfuração ocular traumática. Após sedação com tiletamina e zolazepam para contenção, o animal foi avaliado, sendo detectada lesão não-profunda com comprometimento dérmico e muscular, em plano nasal na região dorsal, sem lesão óssea. Em avaliação oftálmica foi detectada a presença de hifema difuso e estafiloma em região dorso-medial. Frente ao quadro traumático, optou-se por intervenção cirúrgica: A indução anestésica foi realizada com propofol e a manutenção anestésica com isofluorano em vaporizador universal. A lesão em derme-musculatura debridada e suturada. Devido a presença de perfuração corneana optou-se por acessar a câmera anterior a partir da lesão primária com a realização de iridectomia parcial. Após a sutura em córnea foi realizado enxerto pediculado através de retalho obtido da conjuntiva bulbar dorso-medial. No pósoperatório foi administrado betametasona por via subconjuntival e cefovecina por via subcutânea. O paciente foi mantido em recinto sob condições controladas e acompanhado diariamente. Após 15 dias do procedimento cirúrgico foi identificada adequada cicatrização tecidual e manutenção da estrutura ocular, com discreto edema corneano residual, considerado apto ao retorno a vida livre.
\end{abstract}

RESUMO

PALAVRAS-CHAVE: Cirurgia, estafiloma, felino silvestre

\section{OCULAR PERFURATION IN JAGUATIRICA (Leopardus pardalis): CASE REPORT}

\footnotetext{
ABSTRACT

Was rescued one ocelot in the region of Feira de Santana, Bahia, with cutaneous laceration and traumatic ocular perforation caused by trauma. After sedation with tiletamine and zolazepam for restraint the animal was evaluated and nom-deep lesion with dermal and muscular involvement was detected in the nasal plane in the 
dorsal region, without bone lesion. In the ophthalmic evaluation, the presence of diffuse hyphema and staphyloma was identified in the dorsal-medial region. Due to the severity of the lesion, we opted for surgical intervention. Anesthetic induction was performed with propofol and anesthetic maintenance with isofluorane with universal vaporizer. The lesion in the dorsal nasal region was debrided and sutured. Due to the presence of corneal perforation it was decided to access the anterior chamber from the primary lesion with partial iridectomy. After cornea suturing a conjunticval pedicle graft was performed from the dorsal-medial bulbar conjunctiva. Subconjunctival betamethasone and subcutaneously cefovecin were administered as medications for the postoperative period. The patient was kept indoors under controlled conditions and monitored daily. After 15 days of the surgical procedure, adequate tissue healing and maintenance of the ocular structure were identified, with discrete residual corneal edema, considered apt to return to free life. An oncelot was rescued in the region of Feira de Santana, Bahia, presenting cutaneous laceration and traumatic ocular perforation. After sedation using tiletamine associated to zolazepam for chemical restraint, the animal was assessed. Superficial lesion, with dermic and muscle involvement was observed, in nasal plane of the dorsal region, with no bone injury. The ophthalmic exam detected presence of diffuse hyphema and staphyloma in dorso-medial region. Due to the severity of traumatic lesions, surgical approach was elected: anesthetic induction performed with Propofol and maintained with isoflurane, using universal vaporizer. The muscle and cutaneous lesion was debrided and sutured. The access for the anterior chamber was achieved by the primary corneal perforation, executing partial iridectomy. After corneal suturing, a conjunctival pedicle graft was performed using the dorsal-medial bulbar conjunctiva. Subconjunctival betamethasone and subcutaneous cefovecin were administered as medications for the postoperative period. The animal was kept in indoor enclosure, under daily monitored conditions. After 15 days of the surgical procedure, adequate tissue healing and maintenance of the ocular structure were identified, with discrete residual corneal edema, and the patient was considered apt to return to wildlife.

KEYWORDS: Surgery, Sthaphyloma, Wild feline

\section{INTRODUÇÃO}

O manejo clínico-cirúrgico de animais silvestres difere em diversos aspectos do realizado em animais domésticos, sobretudo, devido a necessidade de preservação do bem-estar do animal, mantendo a menor interação como o homem, principalmente no pós-cirúrgico, com o intuito de possibilitar seu retorno à natureza. A jaguatirica (Leopardus pardalis) é um mamífero carnívoro da família Felidae, de tamanho médio (7 a $16 \mathrm{~kg}$ ), que atualmente apresenta estado de conservação considerado pouco preocupante, com distribuição entre no México e o nordeste da Argentina, sendo encontrada em biomas brasileiros (IUCN, 2017).

Enfermidades oculares e anexos em felinos domésticos como a uveíte (PONTES et al., 2006), glaucoma (ORIÁ et al., 2013), endoftalmite (DELGADO, 2015), neoplasias (HESSE et al., 2015) e sequestro corneal (GRAHAM et al., 2017) são descritas na literatura, contudo, foram identificadas poucas descrições sobre enfermidades que cursem com o risco de perda da visão ou estrutura ocular em felinos silvestres, as quais prejudicariam a sobrevivência do animal na natureza, dentre estas catarata em pantera nebulosa (COOLEY, 2001) e em leão do Katanga (STEINMETZ et al., 2005), coloboma (BARNETT ; LEWIS, 2002) e microftalmia em leopardo da neve (HAMOUDI et al., 2013), uveíte por linfoma em lince do deserto 
(AITKEN-PALMER et al., 2011), uveíte facoclastica por infecção por Encephalitozoon cuniculi em leopardo da neve (SCURRELL et al., 2015) e carcinoma sebáceo em tigre siberiano (EDELMANN et al., 2013). Diante do exposto, objetivou-se com o presente trabalho relatar o procedimento anestésico-cirúrgico de uma jaguatirica portadora de perfuração ocular e laceração em face nasal dorsal.

\section{MATERIAL E MÉTODOS}

Uma jaguatirica, fêmea, aproximadamente $10 \mathrm{~kg}$, foi resgatada na região de Feira de Santana, Bahia, ao apresentar laceração cutânea e perfuração ocular, posterior a trauma por arma branca. $\mathrm{O}$ animal foi direcionado a clínica particular (Animalmed®, Feira de Santana, Bahia), onde foi realizada anestesia dissociativa com tiletamina/zolazepam ( $5 \mathrm{mg} / \mathrm{kg}$ ) intramuscular, via dardo, para contenção química. Após o tempo de latência (aproximadamente sete minutos) foi direcionado ao centro cirúrgico.

Observou-se que a lesão em plano nasal, região dorsal, não era profunda, e não houve dano ósseo. No bulbo do olho foi identificado hifema difuso e estafiloma em região dorso-medial (Figura 1A). Optou-se por intervenção cirúrgica, debridamento e sutura da ferida em derme/musculatura e realização de enxerto conjuntival pediculado no olho perfurado. Como protocolo pré-cirúrgico foi instilada uma gota de colírio antibiótico à base de moxifloxacino (Vigamox®, Alcon Laboratórios do Brasil, São Paulo, Brasil) a cada 15 minutos; uma gota de tropicamida (Mydriacyl®, Alcon Laboratórios do Brasil, São Paulo, Brasil) a cada 20 minutos; e uma gota de acetato de prednisolona 1\% (Predfort®, Alcon Laboratórios do Brasil, São Paulo, Brasil). Seguiu-se a indução anestésica com propofol (Propovan ${ }^{\circledR}$, Cristália Produtos Químicos e Farmacêuticos, São Paulo, Brasil) 2 $\mathrm{mg} / \mathrm{kg}$, via intravenosa, e intubação orotraqueal com sonda da Magill ํo 4,5. Para manutenção anestésica foi utilizado isofluorano em circuito Mapleson D, com fluxo de oxigênio $300 \mathrm{~mL} / \mathrm{kg} / \mathrm{min}$ em aparelho dotado de vaporizador universal.

Para correção da lesão em derme e musculatura foi realizada tricotomia, antissepsia com clorexidina em solução aquosa a $0,5 \%$ (solução antisséptica), e debridamento da região lesionada com auxílio de lâmina de bisturi oㅡ 15. A aproximação tecidual foi realizada com fio de mononáilon 3-0 (Nylon, Bioline, Goiânia, Brasil) em padrão simples. Para a realização do enxerto pediculado, a superfície periocular foi tricotomizada e realizada a antissepsia ocular com haste flexível de algodão e solução antisséptica. Iniciou-se o procedimento através de cantotomia temporal. Devido a presença de perfuração corneana com sinéquia anterior optou-se por acessar a câmera anterior a partir da lesão primária. A presença de tecido com aspecto necrótico condicionou a realização de iridectomia parcial, ocorrendo discreto sangramento. A manutenção da patência da câmera anterior foi obtida com o uso de solução salina a $0,9 \%$ em seringa de $3 \mathrm{~mL}$, através de agulha $13 \times 0,45 \mathrm{~mm}$ inserida na região próxima ao limbo. A utilização da solução salina permitiu a remoção do hifema residual.

A córnea foi suturada com fio absorvível multifilamentar sintético (Vicryl® 8-0, Johnson \& Johnson do Brasil, São José dos Campos, Brasil) em padrão simples interrompido. Adicionalmente foi realizado enxerto pediculado através de retalho obtido da conjuntiva bulbar dorso-medial. Foi administrado betametasona (medicamento genérico) $0,5 \mathrm{mLI}$, via subconjuntival e cefovecina (Convênia ${ }^{\circledR}$, Zoetis Indústria de Produtos Veterinários Ltda, São Paulo, Brasil) $8 \mathrm{mg} / \mathrm{kg}$, via subcutânea. 
Finalizado o procedimento, o paciente foi mantido em recinto sob condições monitoradas, de maneira a limitar o contato com seres humanos e possibilitar o acompanhamento diário para verificar a evolução do processo cicatricial e o comportamento do animal em relação ao local da lesão, uma vez que não foi colocado colar protetor.

\section{RESULTADOS E DISCUSSÃO}

Não foi detectada automutilação ou tentativa de atingir a região abordada cirurgicamente. Após 15 dias do procedimento cirúrgico, o animal foi avaliado e identificada adequada cicatrização tecidual e manutenção da viabilidade da estrutura ocular, com discreto edema corneano residual (Figura 1B), considerado apto ao retorno a vida livre.

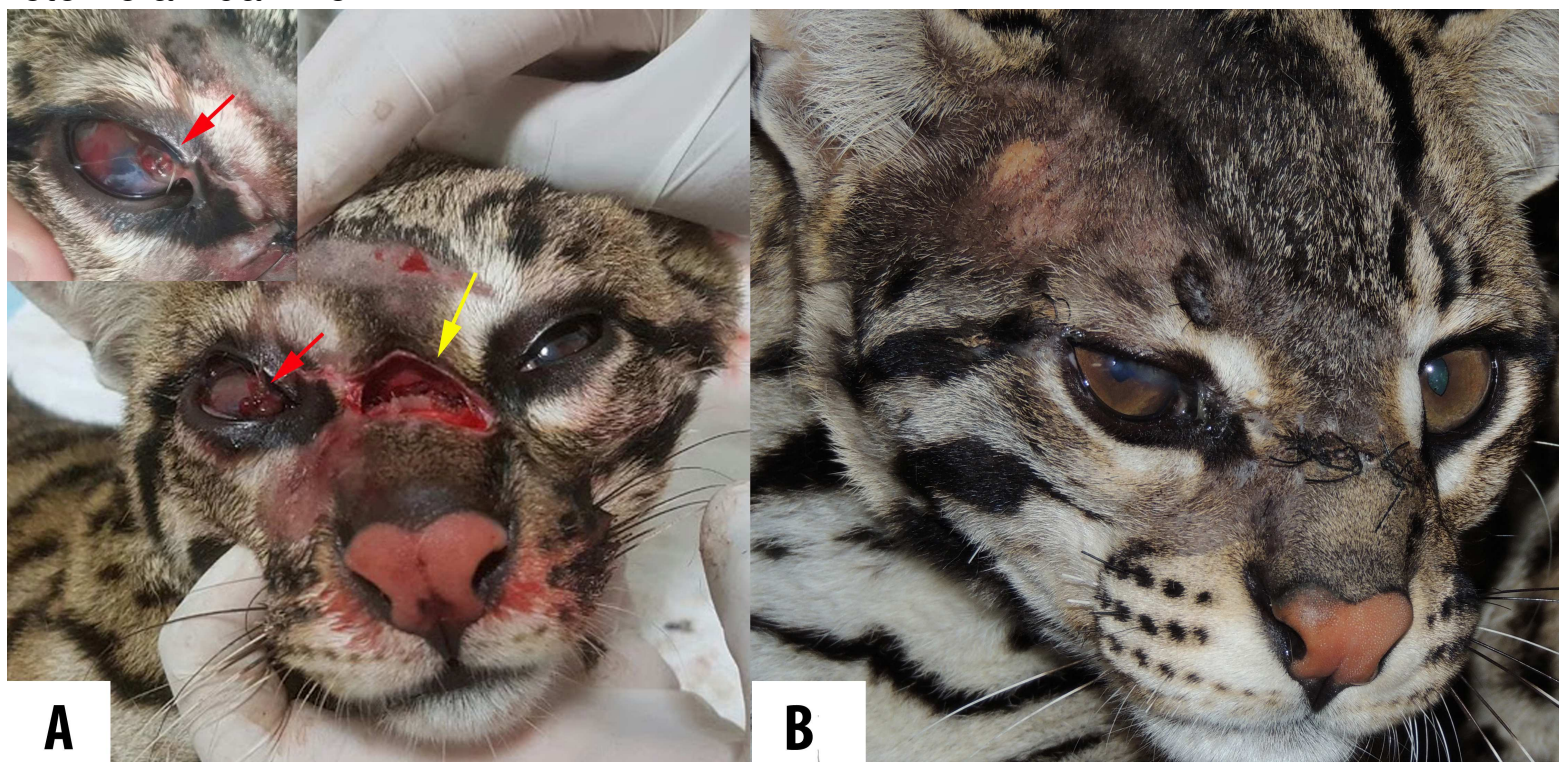

FIGURA 1. Fotografia da face de Leopardus pardalis. A - Notar laceração em região nasal dorsal (seta amarela) e perfuração ocular em olho direito (seta vermelha). Em detalhe notar estafiloma e hifema. B - Aspecto após 15 dias da cirurgia. Notar discreto edema corneano residual em olho direito. Fonte: Arquivo pessoal (2016)

O manejo de felídeos selvagens deve ser realizado de forma cuidadosa devido aos riscos de acidente para o animal e o manipulador da contenção. Comumente é empregada a sedação nestes animais com o uso de dardos, com fármacos de atividade dissociativa, devido a rápida indução e imobilização (SHURRY, 2007).

A anatomia e os princípios gerais da anestesia de felinos exóticos ou silvestres de pequeno porte são similares aos de felinos domésticos e normalmente não são observadas intercorrências (GUNKEL, 2007). Dentre os protocolos descritos em jaguatiricas citam-se, de forma rotineira, a associação de tiletamina e zolazepam (SHINDLE e TEWES 2000; FRANKLIN et al. 2008; NAVA et al. 2008) e cetamina e xilazina (BELTRAN e TEWES 1995; HARVESON et al., 2004; FRANKLIN et al. 2008; RENDÓN-FRANCO et al., 2012).

Optou-se pelo uso da tiletamina e zolazepam pela menor ação hipotensora dessa associação farmacológica quando comparada à cetamina e xilazina, e por ser 
observado que em gatos não apresenta efeito clínico significante sobre a pressão intraocular (YANMAZ et al., 2016) o que é útil em procedimentos intraoculares por reduzir as complicações transoperatórias.

É controversa a utilização de corticosteroides na presença de ulcerações em córnea em animais domésticos, devido à inibição da regeneração epitelial e endotelial e a atividade fibroblástica, fato este que pode progredir com a colagenólise e perfuração ocular (MAGGS, 2013). Sua indicação é direcionada ao pós-cirúrgico de procedimentos intraoculares devido a sua ação anti-inflamatória, e, diante da gravidade do quadro oftálmico e presença de hifema, optou-se no préoperatório pelo uso de corticosteroide por via tópica e ao término da cirurgia por via subconjuntival, de forma a proporcionar absorção farmacológica gradativa e não inibir a agregação plaquetária (TRBOLOVÁ, 2014).

O uso de membrana amniótica (BARROS et al., 2005), pericárdio bovino (DULAURENT et al., 2014) ou enxerto pediculado (GRAHAM et al., 2017) são opções de recobrimentos para amplas lesões corneanas oculares. Salienta-se que o tratamento deve permitir a reconstrução da córnea, manter sua transparência e eixo visual. A principal vantagem do enxerto pediculado é a facilidade e disponibilidade se comparado aos demais materiais doadores (MAGGS, 2013; GRAHAM et al., 2017). O enxerto pediculado proporcionou o recobrimento da lesão e permitiu a vascularização da região, de forma que o uso do antimicrobiano sistêmico de longa ação pode ter alcançado o foco lesional com consequente redução do risco de infecção.

\section{CONCLUSÃO}

O presente trabalho descreveu a realização de abordagem cirúrgica para o recobrimento e melhora na qualidade do manejo terapêutico de Leopardus pardalis de forma a possibilitar a manutenção da estrutura ocular e a preservação da função visual em animal de vida livre o que permitiu a sua reintrodução na natureza.

\section{AGRADECIMENTOS}

A médica veterinária Fernanda Libório, ao médico veterinário Bruno Lemos e a Clínica Animalmed pelo apoio técnico.

\section{REFERÊNCIAS}

AITKEN-PALMER, C.; ISAZA, R.; DUNBAR, M.; BLACKWOOD, S.; GERLACH, T.; RUSSELL, K. Anterior uveitis as an atypical presentation of large granular lymphoma in a caracal (Caracal caracal). Veterinary Ophthalmology, v. 14, n. 5, p. 337-4-, 2011. Disponível em: <http://dx.doi.org/ 10.1111/j.1463-5224.2011.00905.x>. doi: 10.1111/j.1463-5224.2011.00905.x.

BARNETT, K. C.; LEWIS, J. C. M. Multiple ocular colobomas in the snow leopard (Uncia uncia). Veterinary Ophthalmology, v.5, n. 3, p. 197-99, 2002. Disponível em: <http://dx.doi.org/ 10.1046/j.1463-5224.2002.00219.x >. doi: 10.1046/j.14635224.2002.00219.x.

BARROS, P. S. M.; SAFATLE, A. M. V.; GODOY, C. A.; SOUZA, M. S. B.; BARROS, L. F. M.; BROOKS, D. E. Amniotic membrane transplantation for the reconstruction of the ocular surface in three cases. Veterinary Ophthalmology, v. 8, n. 3, p. 189-192, 
2005. Disponível em: <http://dx.doi.org/ 10.1111/j.1463-5224.2005.00391.x >. doi: 10.1111/j.1463-5224.2005.00391.x.

BELTRAN, J.; TEWES, M. Immobilization of ocelots and bobcats with ketamine hydrochloride and xylazine hydrochloride. Journal of Wildlife Diseases, v. 31, p. 4348, 1995. Disponível em: <http://dx.doi.org/ 10.7589/0090-3558-31.1.43 >. doi: 10.7589/0090-3558-31.1.43.

COOLEY, P. L. Phacoemulsification in a clouded leopard (Neofelis nebulosa) Veterinary Ophthalmology, v. 4, n. 2, p. 113-17, 2001. Disponível em: <http://dx.doi.org/ 10.1046/j.1463-5224.2001.00185.x>. doi: 10.1046/j.14635224.2001.00185.x.

DELGADO, E. Endophthalmitis due to an intra-ocular linear foreign in a cat. Journal of Feline Medicine and Surgery Open Reports, v. 1, n. 1, p.1-5, 2015. Disponível em: <http://dx.doi.org/ 10.1177/2055116915585018>. doi: $10.1177 / 2055116915585018$.

DULAURENT, T.; AZOULAY, T.; GOULLE, F.; DULAURENT, A.; MENTEK, M.; PEIFFER, R. L.; ISARD, P. Use of bovine pericardium (Tutopatch $\AA$ ) graft for surgical repair of deep melting corneal ulcers in dogs and corneal sequestra in cats. Veterinary Ophthalmology, v.17, n. 2, p. 91-99, 2014. Disponível em: <http://dx.doi.org/ 10.1111/vop.12047 >. doi: 10.1111/vop.12047.

EDELMANN, M. L.; UTTER, M. L.; KLEIN, L. V.; WOTMAN, K. L. Combined excision and intralesional bevacizumab for sebaceous carcinoma of the eyelid in an Amur tiger (Panthera tigris altaica). Veterinary Ophthalmology, v. 16, n.3, p. 219-224, 2013. Disponível em: <http://dx.doi.org/ 10.1111/j.1463-5224.2012.01051.x. > doi: 10.1111/j.1463-5224.2012.01051.x..

FRANKLIN, S. P.; KAYS, R. W.; MORENO, R.; TERWEE, J. A.; TROYER, J. L.; VANDEWOUDE, $S$. Ocelots on Barro Colorado Island Are Infected with Feline Immunodeficiency Virus but Not Other Common Feline and Canine Viruses. Journal of Wildlife Diseases, v. 44, n. 3, p. 760-765, 2008. Disponível em: <http://dx.doi.org/ 10.7589/0090-3558-44.3.760 >. doi: 10.7589/0090-3558-44.3.760.

GRAHAM, K. L.; WHITE, J. D.; BILLSON, F. M. Feline corneal sequestra: outcome of corneoconjunctival transposition in 97 cats (109 eyes). Journal of Feline Medicine and Surgery, v. 19, n. 6, p.1-7, 2017. Disponível em: <http://dx.doi.org/ https://doi.org/10.1177/1098612X16645144 doi: https://doi.org/10.1177/1098612X16645144.

GUNKEL, C.; LAFORTUNE, M. Felids. In: WEST, G.; HEARD, D.; CAULKETT, N. Zoo animal and wildlife immobilization and anesthesia. Blackwell Publishing: lowa, 2007, p. 443-457.

HAMOUDI, H.; RUDNICK, J.; PRAUSE, J. U.; TAUSCHER, K.; BREITHAUPT, A.; et al. Anterior segment dysgenesis (Peters' anomaly) in two snow leopard (Panthera 
uncia) cubs. Veterinary Ophthalmology, v.16, Supplement 1, p. 130-134, 2013. Disponível em: <http://dx.doi.org/ 10.1111/vop.12017 >. doi: 10.1111/vop.12017.

HARVESON, P. M.; TEWES, M. E.; ANDERSON, G. L.; LAACK, L. L. Habitat use by ocelots in south Texas: implications for restoration. Wildlife Society Bulletin, v. 32, p. 948-54, 2004. Disponível em: <http://dx.doi.org/ 10.2193/0091-7648 >. doi: 10.2193/0091-7648.

HESSE, K. L.; FREDO, G.; GUIMARÃES, L. L. B.; REIS, M. O.; PIGATTO, J. A. T.; et al. Ocular and annexes neoplasms in dogs and cats in Rio Grande do Sul: cases 265 (2009-2014). Pesquisa Veterinária Brasileira, v. 35, n. 1, p.49-54 , 2015. Disponível em: <http://dx.doi.org/ 10.1590/S0100-736X2015000100011>. doi: 10.1590/S0100-736X2015000100011

IUCN 2017. IUCN red list of Threatened Species. Disponível em: <http://dx.doi.org/10.2305/IUCN.UK.2015-4.RLTS.T11509A50653476.en>. Acessado em 13.07.2017.

MAGGS, D. J. Ocular pharmacology and therapeutics. In: MILLER, P. E.; OFRI, $R$. Slatter's fundamentals of veterinary ophthalmology. St Louis: Elsevier Saunders, 5th ed., 2013, pp. 27-59.

NAVA, A. F. D.; CULLEN JR, L.; SANA, D. A.; NARDI, M. S.; RAMOS FILHO, J. D.; et al. First Evidence of Canine Distemper in Brazilian Free-Ranging Felids. EcoHealth, v. 5, p. 513-18, 2008 Disponível em: <http://dx.doi.org/ 10.1007/s10393008-0207-8 >. doi: 10.1007/s10393-008-0207-8.

ORIÁ. A.P.; GOMES JUNIOR, D. C.; SOUZA, M. R.; COSTA NETO, J. M.; ESTRELA LIMA, A.; DÓREA NETO, F. A. Glaucoma secundário em cães e gatos. Medicina Veterinária, v.7, n. 3, p. 13-22, 2013.

PONTES, K. C. S.; VIANA, J. A.; DUARTE, T. S. Etiopatogenia da uveíte associada a Doenças infecciosas em pequenos animais. Revista ceres, v. 53, n. 309, p. 61826, 2006.

RENDÓN-FRANCO, E.; CASO-AGUILAR, A.; JIMENÉZ-SÁNCHEZ, N. G.; HERNANDEZ-JAUREGUI, D. M. B.; SANDOVAL-SÁNCHEZ, A. L.; ZEPEDALÓPEZ, H. M. Prevalence of Anti-Toxoplasma gondii Antibody in Free-ranging Ocelots (Leopardus pardalis) from Tamaulipas, Mexico. Journal of Wildlife Diseases, v. 48, n.3, p.829-31. Disponível em: <http://dx.doi.org/ 10.7589/00903558-48.3.829 >. doi: 10.7589/0090-3558-48.3.829.

SCURRELL, E. J.; HOLDING, E.; HOPPER, J.; DENK, D.; FUCHSBAUMGARTINGER, A.; et al. Bilateral lenticular Encephalitozoon cuniculi infection in a snow leopard (Panthera uncia). Veterinary Ophthalmology, v.18, Supplement 1, p. 143-147, 2015. Disponível em: <http://dx.doi.org/ 10.1111/vop.12218>. doi: $10.1111 /$ vop. 12218. 
SHINDLE, D. B.; TEWES, M. E. Immobilization of wild ocelots with tiletamine and zolazepam in southern Texas. Journal of Wildlife Diseases, v.36, p. 546-50, 2000. Disponível em: <http://dx.doi.org/ 10.7589/0090-3558-36.3.546>. doi: 10.7589/00903558-36.3.546.

SHURRY, T. Capture and Physical Restraint of Zoo and Wild Animals. In: WEST, G.; HEARD, D.; CAULKETT, N. Zoo animal and wildlife immobilization and anesthesia. Blackwell Publishing: lowa, 2007, p. 131-144.

STEINMETZ, A.; EULENBERGER, K.; THIELEBEIN, J.; BUSCHATZ, S.; BERNHARD, A.; et al. Cataracts and other ophthalmic findings in a group of closely related Angola lions (Panthera leo bleyenberghi). In: Abstracts: European College of Veterinary Ophthalmologists and European Society of Veterinary Ophthalmology, Oporto, Portugal, 15-19 June 2005, Veterinary Ophthalmology, n. 8, n.6, p. 42736, 2005.

TRBOLOVÁ, A. Using of corticosteroids in small animals ophthalmology. e-Polish Journal of Veterinary Ophthalmology, v.1, p. 1-8, 2014.

YANMAZ, L. E.; DOGAN, E.; OKUR, S.; OKUMUS, Z.; ERSOZ, U. Comparison of the effects of intranasal and intramuscular administrations of zolazepam-tiletamine combination on intraocular pressure in cats Latif Emrah. Veterinary Ophthalmology Supplement 1, p. 115-8, 2016. Disponível em: <http://dx.doi.org/ 10.1111/vop.12400 >. doi: 10.1111/vop.12400. 